\title{
Comments on Pinched Hysteresis Loops of Memristive Elements
}

\author{
Zdenek BIOLEK ${ }^{1}$, Dalibor BIOLEK ${ }^{1,2}$, Viera BIOLKOVA ${ }^{3}$, Zdenek KOLKA ${ }^{3}$ \\ ${ }^{1}$ Dept. of Microelectronics, Brno University of Technology, Technicka 10, 61600 Brno, Czech Republic \\ ${ }^{2}$ Dept. of Electrical Engineering, University of Defense, Kounicova 65, 66210 Brno, Czech Republic \\ ${ }^{3}$ Dept. of Radio Electronics, Brno University of Technology, Technicka 12, 61600 Brno, Czech Republic \\ zdenek.biolek@gmail.com, dalibor.biolek@unob.cz, biolkova@feec.vutbr.cz, kolka@feec.vutbr.cz
}

\begin{abstract}
The hysteresis loops pinched in the $v$-i origin belong to well-known fingerprints of memristive elements driven by bipolar periodical signals. Some element properties follow from the loop behavior in the close neighborhood of the origin. The paper analyzes this behavior of the memristive elements that produce steady-state hysteresis loops under harmonic excitation. It is shown that there is a connection between the frequency content of the state variable waveform and the type of the loop being pinched.
\end{abstract}

\section{Keywords}

Memristive element, memristance, pinched hysteresis loop, crossing-type, tangential pinching

\section{Introduction}

In 1976, the class of the so-called memristive elements was defined [1]. Such elements behave as nonlinear resistors whose resistances, referred to as memristances, depend on the state $x$ of an associated dynamical system according to Ohm's law [1]

$$
v=R_{\mathrm{M}}(x, i) i .
$$

Here $v$ and $i$ are the terminal voltage and current of the element, and $R_{\mathrm{M}}$ is the memristance, expressing the memory effect caused by the state dynamics, governed by the equation of motion

$$
\frac{d x}{d t}=f(x, i) .
$$

Since the state $x$ can also be of non-electric nature, the memristance $R_{\mathrm{M}}$ becomes a universal measure for assessing memory symptoms, which have recently come to be explored across the assorted physical and biological platforms [2-5]. A specific memristive element is the memristor introduced in 1971 in [6], where $R_{\mathrm{M}}(x, i)=R_{\mathrm{M}}(q)$, thus the charge $q=\int i d t$ serves as its state variable.

The discovery of the so-called HP memristor [7] initiated and accelerated increased interest in the theory of memristive systems. Experiments have revealed that the memristance can be the consequence of a broad spectrum of phenomena of various physical nature. As one of the results of such research, the memristance is generalized as a function of a vector state $\boldsymbol{x}$. The element, complying with (1) and (2), where the state variable can be of either scalar or vector form, is currently denoted the extended memristor [8]. The memristor originally introduced in [6] is now regarded as the ideal memristor [8].

The memristance, regardless of its physical origin, stamps its specific fingerprint, the pinched hysteresis loop, in the current-voltage characteristic. For passive memristors, zero-valued instantaneous voltage always corresponds to zero-valued instantaneous current and vice versa, and the $v-i$ characteristic must cross the $v-i$ origin. Since the memristance is state-dependent, the characteristic has generally a different slope when the operating point leaves the origin than if it comes back. As a result, the $v-i$ characteristic is ambiguous and dependent on the type of excitation. On the other hand, it has always the character of a loop pinched at the origin.

In [3], it is pointed out for the first time that the loop behavior in the close neighborhood of the origin provides data for examining the nature of the memory that is associated with the memristive element. It is suggested that the loops be classified as type-I and type-II loops. For type I (II), the loop arms (do not) intersect at the origin. It was proved in [9] that the loops of the ideal memristor, which can be considered a prototype of the nonvolatile memory, are always of type I. On the other hand, inexhaustible types of volatile memory elements exhibit the type-II loops. Let us mention incandescent bulbs, fluorescent tubes, glowlamps, etc. [10]. However, it has not hitherto been proved whether there are some connections between the loop type and the volatility of the element, and no universal criterion is available for evaluating the type of the crossing according to the type of the functions $R_{\mathrm{M}}()$ and $f()$ from (1) and (2).

Examples of various loops are given in Fig. 1. The loops (a) and (c) are of type I, the loop (b) is of type II.

A more detailed classification of the loop passing through the origin has been done in [11]. The loop passing is of the Crossing-Type (CT) or Non-Crossing Type (NCT, 


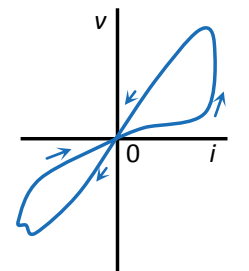

(a)

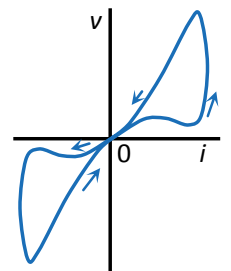

(b)

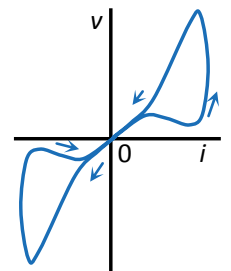

(c)
Fig. 1. Examples of the hysteresis loops: (a) $\mathrm{CT}(0)$, (b) $\operatorname{NCT}(1),(c) \operatorname{CT}(2)$.

also referred to as tangential type), depending on whether the two arms of the loop intersect or only touch, and the order of touching [11] is analyzed in the latter case. The touching of the two arms is indicated by equal derivatives of the functions $v^{+}(i)$ and $v^{-}(i)$, which approximate the loop arms near the origin. The positive arm (labeled by the superscript + ) is the arm followed by the operating point passing from the first to the third quadrant. All the varieties of possible passing through the origin can be expressed by a combination of two specifications: the CT/NCT, and the order of touching. The analysis of the type of the loop pinching is based on the successive evaluation of the formula

$$
\Delta^{(k)}=\lim _{i \rightarrow 0}\left(\frac{d^{k}}{d i^{k}}\left(v^{+}(i)-v^{-}(i)\right)\right)
$$

for $k=1,2, . .$, as illustrated in Fig. 2 .

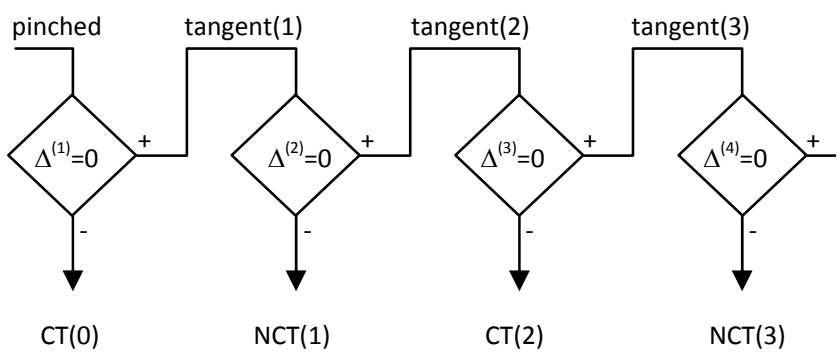

Fig. 2. Classification of loop pinching. Adopted from [11].

The derivatives of both arms for $v=0$ and $i=0$ are gradually compared as long as they are identical. The order of touching is then equal to the highest order of the identical derivatives. As a consequence, the order of touching 0 denotes the case when the loop arms intersect. It is obvious from Fig. 2 that the CT loop must always be of even-order touching, and the NCT loop must be of oddorder touching.

It is proved in [11] for the ideal memristors, driven by a periodical signal as an odd function of time, that the NCT loops, i.e. with odd-order of touching, are impossible. Similar regularities for general memristive elements according to (1) and (2) have not yet been published. This paper analyzes possible types of the loops generated by the above systems under their excitation by a sinusoidal current. We focus on elements whose dynamics (2) respond to the sinusoidal excitation $i(t)$ by a steady-state periodical waveform $x(t)$. Section 2 describes the derivation of concrete forms of formula (3) for $k=1,2$, and for an arbitrary excitation $i(t)$, and formulates the conditions for the CT( 0$)$ and NCT loops. Based on these results, the general regularities of passing through the $v-i$ origin for sinusoidal driving current are stated in Sec. 3. Section 4 illustrates the above results via the SPICE simulation of the electrical wire, which as a power-controlled temperature-dependent resistor fulfills the conditions defining the memristive element.

\section{Evaluation of $\Delta^{(1)}$ and $\Delta^{(2)}$}

Evaluating (3) is the key to revealing the type of a loop pinching according to Fig. 2. Differentiating (1), the loop slope at a point that coincides with the operating point can be derived as

$$
\frac{d v}{d i}=R_{\mathrm{M}}(x, i)+\frac{d R_{\mathrm{M}}(x, i)}{d i} i .
$$

The successive differentiation of (4) yields

$$
\frac{d^{k} v}{d i^{k}}=k \frac{d^{k-1} R_{\mathrm{M}}(x, i)}{d i^{k-1}}+\frac{d^{k} R_{\mathrm{M}}(x, i)}{d i^{k}} i .
$$

Let us demonstrate the procedure of specifying (3) for $k=1$ and 2 . Note that the second right-hand side terms of (4) and (5) are zero at the $v$-i origin. It holds for $k=1$ :

$$
\Delta^{(1)}=R_{\mathrm{M}}\left(x^{+}, 0\right)-R_{\mathrm{M}}\left(x^{-}, 0\right)
$$

where $x^{+}$and $x^{-}$are the values of the state $x$ at time instants when the operating point comes to the origin along the arms $v^{+}$and $v^{-}$, respectively.

For $k=2$ we can conclude that

$$
\frac{d^{2} v}{d i^{2}}=2 \frac{d R_{\mathrm{M}}(x, i)}{d i}+\frac{d^{2} R_{\mathrm{M}}(x, i)}{d i^{2}} i .
$$

The second right-hand side term in (7) is zero at the origin. The first term can be modified to the following form:

$$
2 \frac{d R_{\mathrm{M}}(x, i)}{d i}=2 \frac{\partial R_{\mathrm{M}}(x, i)}{\partial x} \frac{d x}{d i}+2 \frac{\partial R_{\mathrm{M}}(x, i)}{\partial i} .
$$

The state equation (2) can help to obtain the identity

$$
\frac{d x}{d i}=\frac{d x}{d t} \frac{d t}{d i}=\frac{f(x, i)}{\frac{d i}{d t}}
$$

Then $\Delta^{(2)}$ can be written in the form

$$
\Delta^{(2)}=2\left(\frac{\partial R_{\mathrm{M}}^{+}}{\partial x} \frac{f^{+}}{\frac{d i^{+}}{d t}}-\frac{\partial R_{\mathrm{M}}^{-}}{\partial x} \frac{f^{-}}{\frac{d i^{-}}{d t}}\right) .
$$


The superscripts - and + denote the values of the corresponding functions at the beginning and the end of drawing the loop lobe in the first quadrant.

It is obvious from (6) that only the instantaneous values of the memristance determine whether the arms of the loop will touch or not. It follows from (10) that the order of touching is also affected by the instantaneous rate of the changes of the driving current.

\section{Loop Classification according to the Waveform of State $x(t)$}

The definition of resistive element (1) controlled by the state of a general dynamical system with scalar state (2) can include elements with arbitrary complex dynamics. For example, an associated dynamical system governed by (2) might be unstable. Then there would be no steady-state response to the periodical excitation, and the pinched hysteresis loops would not converge to steady pattern in the $v-i$ plane. A different complication would be associated with the prospective sub-harmonic oscillations in the steadystate response. Then only a part of the hysteresis loop would be drawn within one period of the driving signal. In order to prevent the above troubles, let us confine ourselves to elements that fulfill the following assumption:

There is such an initial state $x_{0}$ for which and for the sinusoidal driving function

$$
i(t)=I_{\mathrm{MAX}} \sin (\omega t)
$$

the solution of differential equation (2) converges to a periodical steady-state solution $x(t)$ with the repeating frequency $\omega_{x}=n \omega$, and $I_{\mathrm{MAX}}, \omega=2 \pi / T$, and $T$ as the amplitude, angular frequency, and repeating period of the driving signal; $n$ is a positive integer.

The purpose of this assumption is to warrant the existence of the steady state, when the same pinched hysteresis loop is redrawn within each repeating period of the driving signal.

Let us prove below that the type of the loop pinching is determined by whether the ratio $n$ of the repeating frequencies of the response $x(t)$ to the excitation (11) is an odd or even number. It is well-known that $n=1$ for ideal memristors [9], because their state variable is the charge $q$, whose frequency is equal to the frequency of current. The steady state is established immediately, and the pinching is of the crossing type (CT). On the other hand, $n=2$ for thermistors [1] and similar power-driven memristive elements, and their steady-state loops are of the non-crossing types (NCT).

For $n$ odd, the states $x^{+}$and $x^{-}$will be generally different. Then also the memristance $R_{\mathrm{M}^{+}}=R_{\mathrm{M}}\left(x^{+}, 0\right)$ and $R_{\mathrm{M}-}=R_{\mathrm{M}}\left(x^{-}, 0\right)$ and the initial and final slopes of the loop lobe will differ. This means that $\Delta^{(1)} \neq 0$, and the following partial conclusion can be stated:

If the frequency spectrum of the periodical response of the state $x(t)$ starts with a harmonic component whose frequency is an odd multiple of the frequency of the sinusoidal excitation, then the native behavior of the loop is CT( 0$)$.

One exception to this rule is the singular case when the state $x$ is the same at the beginning and in the middle of the period. Another exception holds if the memristance is the same at the beginning and in the middle of the period, although the states at these time instants are different. This may happen, for example, if the resistance $R_{\mathrm{M}}(x, 0)$ is not a monotonic function of the state, and at the beginning and in the middle of the period the operating point coincidentally comes to two states which cause identical memristances. Then the other conditions according to Fig. 2 determine the type of pinching. For the sinusoidal excitation (11), equation (10) for $\Delta^{(2)}$ has the concrete form

$$
\Delta^{(2)}=-\frac{2}{\omega I_{\mathrm{MAX}}}\left(\frac{\partial R_{\mathrm{M}}^{+}}{\partial x} f^{+}+\frac{\partial R_{\mathrm{M}}^{-}}{\partial x} f^{-}\right) .
$$

The equality $f(q, i)=i$ holds for the ideal memristor. This means that $f^{+}=f^{-}=0$, so that $\Delta^{(2)}=0$, and the case NCT(1) is foreclosed according to Fig. 2. This conclusion is comfortable with the proof in [11] that all NCT points are excluded for ideal memristors regardless of the order of touching. For cases when the memristances are equal at the beginning and in the middle of the period, both arms of the loop will exhibit even-order touching at the origin (of the $2^{\text {nd }}$ order at the least).

Let us now analyze the case when $n$ is an even integer. Then the equality $x^{+}=x^{-}$holds, which implies $R_{\mathrm{M}^{+}}=R_{\mathrm{M}^{-}}, \Delta^{(1)}=0$, and also $f^{+}=f^{-}, \partial R_{\mathrm{M}^{+}} / \partial x=\partial R_{\mathrm{M}-} / \partial x$, and finally

$$
\Delta^{(2)}=-\frac{4}{\omega I_{\mathrm{MAX}}} \frac{\delta R_{\mathrm{M}}^{+}}{\delta x} f^{+} .
$$

It follows from (13) that, except the singular case when $\left(\partial R_{\mathrm{M}^{+}} / \partial x\right) \vee\left(f^{+}=0\right)$, the inequality $\Delta^{(2)} \neq 0$ holds. We can conclude the following:

If the frequency spectrum of the periodical response of the state $x(t)$ starts with a harmonic component whose frequency is an even multiple of the frequency of the sinusoidal excitation, then the native behavior of the loop is $\mathrm{NCT}(1)$.

For the case of the above exception, it is necessary to specify the type of pinching via the additional procedure described in Fig. 2.

\section{Illustrative Example}

It is well known that temperature-dependent resistors such as thermistors, bulbs, or a simple wire [12] belong to 
memristive elements [1], which exhibit the $v-i$ pinched hysteresis loops under sinusoidal excitation. The main conclusions from Sec. 3 are verified below via the following simulations.

Let us analyze the $v-i$ characteristic of a wire, which is warming up via a passing current of sinusoidal waveform (11). Consider that this wire with the known heat capacity $C_{\mathrm{T}}$ is bounded with the ambient environment with a temperature $T_{\mathrm{amb}}$ via a thermal resistance $R_{\mathrm{T}}$. For a temperature $T_{\text {ref }}$, the wire provides its electrical resistance $R_{\text {ref }}$. Then the memristance from (1) is in the form

$R_{\mathrm{M}}(T)=$

$=R_{\text {ref }}\left(1+\alpha\left(T-T_{\text {ref }}\right)+\beta\left(T-T_{\text {ref }}\right)^{2}+\gamma\left(T-T_{\text {ref }}\right)^{3}\right)$

where $\alpha, \beta$, and $\gamma$ are the linear, quadratic, and cubic temperature coefficients, and the temperature $T$ is the element state.

The temperature dependence of the wire resistance is shown in Fig. 3. The curve holds for $\alpha=4.3 \mathrm{mK}^{-1}, \beta=$ $-55 \mu \mathrm{K}^{-2}, \gamma=150 \mathrm{nK}^{-3}, T_{\text {ref }}=21^{\circ} \mathrm{C}$, and $R_{\text {ref }}=134 \mathrm{~m} \Omega$. The local maximum and minimum appear at temperatures of $70.2^{\circ} \mathrm{C}$ and $216.8^{\circ} \mathrm{C}$, respectively.

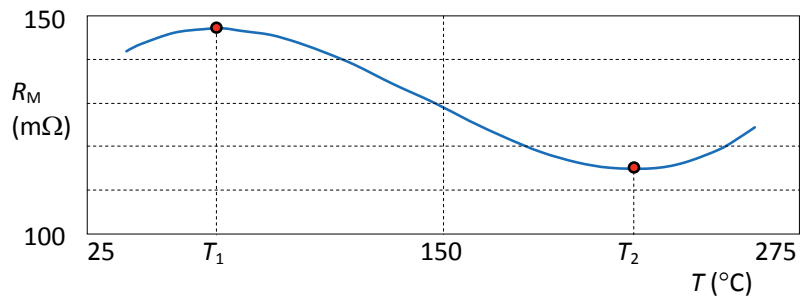

Fig. 3. Temperature dependence of the wire resistance.

The state equation (2) is in the form [12]

$$
\frac{d T}{d t}=\frac{1}{C_{\mathrm{T}}}\left(R_{\mathrm{M}}(T) i^{2}-\frac{T-T_{\mathrm{amb}}}{R_{\mathrm{T}}}\right) .
$$

Figure 4 shows the results of a simulation which was conducted under the following conditions: sinusoidal $8 \mathrm{~A} / 0.1 \mathrm{~Hz}$ driving current, ambient temperature $T_{\text {amb }}=27^{\circ} \mathrm{C}$, heat capacity $C_{\mathrm{T}}=68.9 \mathrm{~mJ} / \mathrm{K}$.

The curves represent the steady-state waveforms of the resistance of the wire and its temperature within one repeating period of excitation. The versions (a) to (c) hold for three different thermal resistances between the wire and its environment. Different resistances cause different intensities of cooling the wire and thus its different steady-state temperature levels.

The hysteresis loops for all three versions are given in Fig. 5. For the sake of lucidity, the loops (a) and (c) are shifted vertically. The loop (b) is of NCT(1), and this type is native for such an element.

The versions (a) and (c) are selected such that the wire temperatures at time instants when the current excita-

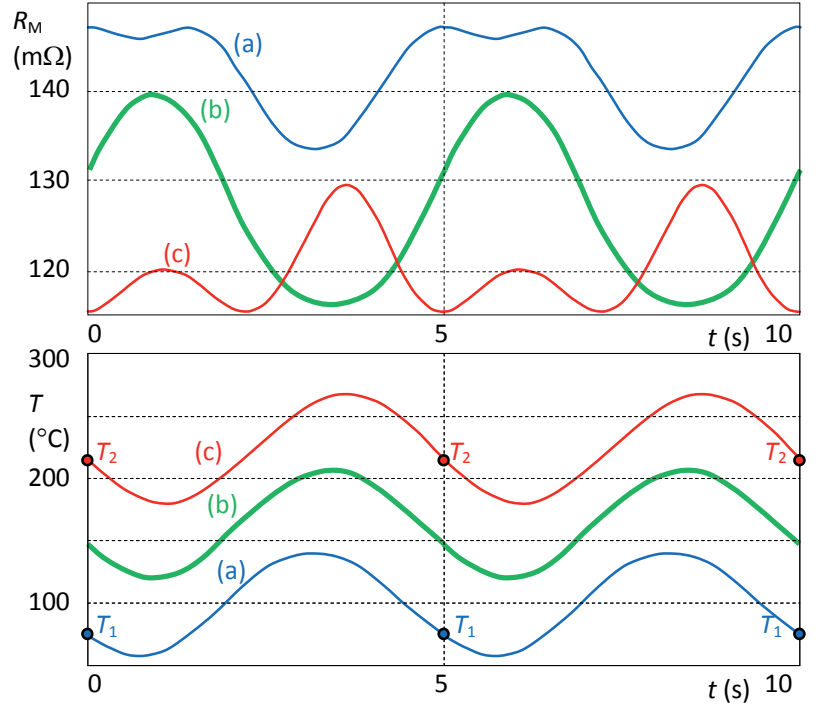

Fig. 4. Steady-state waveforms of the wire resistance and temperature for different thermal resistances $R_{\mathrm{T}}=$ (a) $15.50 \mathrm{KW}^{-1}$, (b) $33.75 \mathrm{KW}^{-1}$, (c) $51.25 \mathrm{KW}^{-1}$.

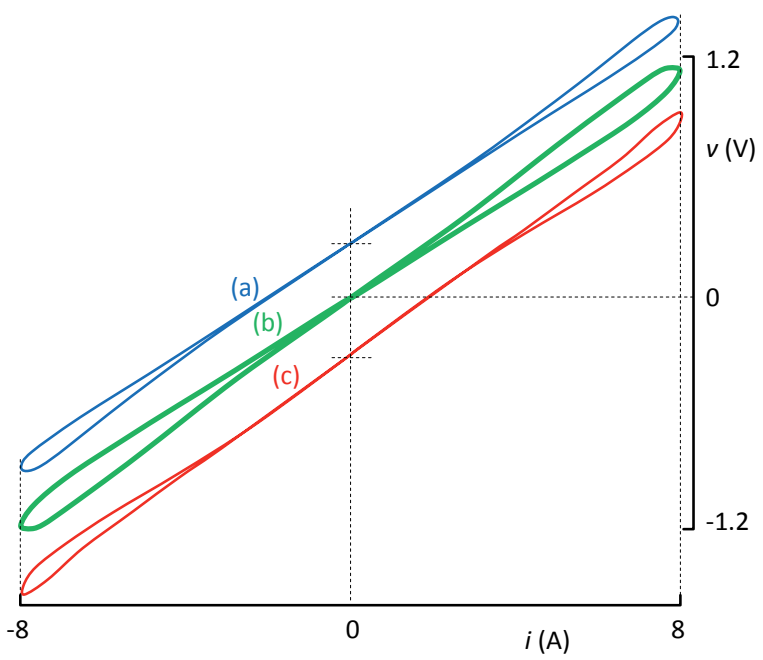

Fig. 5. Pinched hysteresis loops of $\mathrm{NCT}(3)$ : (a), (c), and NCT(1): (b)

tion is zero are exactly equal to the temperatures $T_{1}$ and $T_{2}$ of the local extremes of the function $R_{\mathrm{M}}(T)$. According to (13), $\Delta^{(2)}=0$ for the cases (a) and (c), and it follows from Fig. 2 that such loops cannot be of NCT(1). Since they are not of $\mathrm{CT}(2)$ either, they must be $\mathrm{NCT}(n)$ loops, where $n$ is an odd integer, $n \geq 3$. Comparing the loops in Fig. 5 , it is evident that the loop arms in the cases (a) and (c) exhibit higher-order touching at the origin than in the case (b).

\section{Conclusions}

The paper is a follow-up to the results of researching the pinched hysteresis loops of ideal memristors published in [11]. The methodology of the classification of the loops from [1] was used in this work for studying more general 
memristive systems. However, it is much more difficult to classify a concrete memristive element in terms of such a methodology than ideal memristors. The reason is that the general memristive elements can exhibit incomparably more complex dynamics of their internal states, as well as nonlinearities of their memristances in respect of the driving quantities. From the practical point of view, this can mean that, in contrast to ideal memristors, we must wait until the hysteresis loop becomes stabilized. What makes it even more involved is that the steady state may never been established, for example, if the associated dynamical system is unstable. That is why the analysis is limited in this work to memristive elements that respond to sinusoidal excitation by periodical steady-state motion of the state variable, with the fundamental frequency being an integer multiple of the excitation frequency. Whether this multiple is an even or an odd number can be decisive for the nature of the loop: The even multiples are natively associated with the non-crossing type loops with first-order touching, NCT(1), whereas the odd multiples with the crossing-type loops, thus with zero-order touching CT(0). Exceptions to these rules are also described, which can appear owing to an accidental concurrence of the element parameters and the instantaneous process of the state variable. The mechanism of these exceptions is subject to the classification key in Fig. 2.

Since the temperature-dependent resistive elements are power-controlled elements, they respond to sinusoidal current excitation via doubling the frequency of the state waveform in comparison with the driving frequency. Computer simulations of electric wire driven by sinusoidal current are in full agreement with the theoretical conclusions.

The analyses have been performed for the currentcontrolled memristive elements. Analogous conclusions can be drawn also for the dual case of voltage-controlled memristive elements.

\section{Acknowledgments}

The research is a part of the COST Action IC1103 and it is financially supported by the Czech Ministry of Education under grant no. LD14103. The research was also supported by the Project for the development of K217 Department, UD Brno. Research described in this paper was financed by the Czech Ministry of Education in frame of the National Sustainability Program under grant LO1401. For research, infrastructure of the SIX Center was used.

\section{References}

[1] CHUA, L. O., KANG, S. M. Memristive devices and systems. Proceedings of the IEEE, 1976, vol. 64, no. 2, p. 209-223. DOI: 10.1109/PROC.1976.10092
[2] PERShIN, Y. V., LA FONTAINE, S., DI VENTRA, M. Memristive model of amoeba's learning. Physical Review E, 2009, vol. 80, no. 2, p. 1-6. DOI: 10.1103/PhysRevE.80.021926

[3] PERSHIN, Y. V., DI VENTRA, M. Memory effects in complex materials and nanoscale systems. Advances in Physics, 2011, vol. 60, no. 2, p. 145-227. DOI: 10.1080/00018732.2010.544961

[4] MARTINSEN, Ø. G., GRIMNES, S., LÜTKEN, C. A., JOHNSEN, G. K. Memristance in human skin. Journal of Physics: Conference Series, 2010, vol. 224, no. 1, 012071. DOI: 10.1088/1742-6596/224/1/012071

[5] VOLKOV, A. G., TUCKET, C., REEDUS, J., VOLKOVA, M. I., MARKIN, V. S., CHUA, L. O. Memristors in plants. Plant Signaling \& Behavior, 2014, vol. 9, no. 3, p. 1-8. DOI: $10.4161 /$ psb. 28152

[6] CHUA, L. O. Memristor - The missing circuit element. IEEE Transactions on Circuit Theory, 1971, vol. CT-18, no. 5, p. 507 to 519. DOI: 10.1109/TCT.1971.1083337

[7] STRUKOV, D. B., SNIDER, G. S., STEWART, D. R., WILLIAMS, R. S. The missing memristor found. Nature, 2008, vol. 453, p. 80-83. DOI: 10.1038/nature06932

[8] CHUA, L. O. If it's pinched it's a memristor. Semiconductor Science and Technology, 2014, vol. 29, no. 10, p. 1-42. DOI: 10.1088/0268-1242/29/10/104001

[9] BIOLEK, D., BIOLEK, Z., BIOLKOVÁ, V. Pinched hysteresis loops of ideal memristors, memcapacitors and meminductors must be 'self-crossing'. Electronics Letters, 2011, vol. 47, no. 25, p. 1385-1387. DOI: 10.1049/el.2011.2913

[10] PRODROMAKis, T., TOUMAZOU, CH., CHUA, L. O. Two centuries of memristors. Nature Materials, 2012, vol. 11, p. 478 to 481. DOI: $10.1038 / \mathrm{nmat} 3338$

[11] BIOLEK, Z., BIOLEK, D. How can the hysteresis loop of the ideal memristor be pinched? Circuits and Systems II: Express Briefs, 2014, vol. 61, no. 7, p. 491-495. DOI: 10.1109/TCSII.2014. 2327303

[12] BIOLEK, Z., BIOLEK, D., VÁVRA, J., BIOLKOVÁ, V., KOLKA, Z. The simplest memristor in the world. Submitted to International Conference on Circuits and Systems (ISCAS 2016). Montreal (Canada), 2016.

\section{About the Authors ...}

Zdeněk BIOLEK was born in Ostrava, Czech Republic, in 1959. He received the $\mathrm{PhD}$. degree in Electronics and Informatics from Brno University of Technology, Czech Republic, in 2001.

He is currently with the Department of Microelectronics, Brno University of Technology (BUT), Czech Republic. Until the year 1993 he worked as an independent researcher in semiconductor company TESLA Rožnov. He is the author of unique electronic instruments associated with IC production and testing. He is also the author of several papers from the area of the utilization of variational principles in theoretical electrical engineering and stability testing of resistive circuits, and also from the field of memristors and mem-systems. Dr. Z. Biolek is also the co-author of two books about memristive systems and PSpice modeling and simulation of special electronic circuits including switched-capacitor filters, switched DC-DC converters, and memristors. 
Dalibor BIOLEK received the M.Sc. degree in Electrical Engineering from Brno University of Technology, Czech Republic, in 1983, and the PhD. degree in Electronics from the Military Academy Brno, Czech Republic, in 1989, focusing on algorithms of the symbolic and numerical computer analyses of electronic circuits with a view to the linear continuous-time and switched filters.

He is currently with the Dept. of EE, University of Defense Brno (UDB), and with the Dept. of Microelectronics, Brno University of Technology (BUT), Czech Republic. His scientific activity is directed to the areas of general circuit theory, frequency filters, mem-systems, and computer simulation of electronic systems. He has published over 400 papers and is author of several books on circuit analysis and simulation. At present, he is a professor at BUT and UDB in the field of Theoretical Electrical Engineering.

Prof. Biolek is a member of the CAS/COM Czech National Group of IEEE. He also serves as an Area Editor of the International Journal of Electronics and Communications (AEU).
Viera BIOLKOVÁ was born in Partizánske, Slovakia. She received her MSc. degree in Electrical Engineering from Brno University of Technology, Czech Republic, in 1983. She joined the Department of Radio Electronics in 1985, and is currently working as a Research Assistant at the Department of Radio Electronics, Brno University of Technology (BUT), Czech Republic. Her research and educational interests include signal theory, analog signal processing, and digital electronics.

Zdeněk KOLKA received the M.Sc. degree in 1992 and the Ph.D. degree in 1997, both in Electrical Engineering, from Brno University of Technology. In 1995 he joined the Department of Radio Electronics, Brno University of Technology. His scientific activity is directed to the areas of general circuit theory, computer simulation of electronic systems and digital circuits. For years he has been engaged in algorithms of the symbolic and numerical computer analysis of electronic circuits. He has published over 100 papers. At present, he is a professor at BUT in the field of Radio Electronics. 